\title{
Anisotropic Periodic Assemblies and Metamaterials for Applications to Antennas and Microwave Devices
}

\author{
John L. VOLAKIS ${ }^{\dagger a)}$, Nonmember, Gokhan MUMCU ${ }^{\dagger}$, Student Member, and Kubilay SERTEL ${ }^{\dagger}$, Nonmember
}

SUMMARY Basic microwave properties of magnetic photonic (MPC) and degenerate band edge (DBE) crystals are investigated mathematically and experimentally. Two dimensional and three dimensional models are considered demonstrating the very high sensitivity and field growth associated with these crystals. A major part of the paper deals with the development of realistic anisotropic periodic structures using a combination of layers constructed from thin film frequency selective surfaces, alumina, titanate and calcium vanadium garnet (CVG) materials. Measurements for antenna applications demonstrate and validate the theoretical performance of the MPC and DBE crystals. The latter part of the paper will present an exciting and promising development relating to microwave circuit applications. Specifically, a novel dual-line printed circuit is presented to emulate propagation in anisotropic media. As such, the MPC and DBE phenomena can be realized using very simple printed circuits (coupled lines). Lastly, physically small printed antennas and arrays based on the coupled transmission lines are presented.

key words: metamaterials, anisotropic media, photonic crystals, electromagnetic band gap, degenerate band edge

\section{Introduction}

Controllable dispersion properties of periodic material assemblies, periodic circuit elements and microwave components have attracted considerable attention over the last decade [1]. Left handed or negative index materials and electromagnetic band gap assemblies are among the most popular in this respect, and applications relating to these have been proposed [2], [3].

Apart from the negative index media, a new class of layered periodic assemblies made up of misaligned anisotropic (dielectric and ferromagnetic) layers were shown to give rise to frozen propagation modes [4]. These magnetic photonic crystals (MPCs) have the key properties of (i) coupling the incident electromagnetic energy into the material with minimal reflection, and (ii) subsequently slowing down the field (frozen mode) to give rise to a built-up energy inside the crystal. This interesting aspect of propagation within the MPCs was numerically demonstrated in [5] and shown to lead directive radiation from embedded electrically small sources in [6].

Lack of low-loss ferromagnetic materials for MPC realization led to the degenerate band edge (DBE) assemblies (constructed by removing the ferromagnetic layers from the assembly) and the associated DBE modes [7], [8]. Although DBE assemblies can be manufactured using existing mate-

\footnotetext{
Manuscript received May 13, 2007.

†The authors are with the ElectroScience Laboratory at Ohio State University, 1320 Kinnear Rd, Columbus, OH 43212, USA.

a) E-mail: volakis.1@ osu.edu

DOI: 10.1093/ietcom/e90-b.9.2203
}

rials (such as single crystal rutile: $\mathrm{TiO}_{2}$ layers) the design and manufacturing/testing loop turns out to be rather difficult due to multiple disciplines involved (electrical, materials, and manufacturing). To alleviate this and speed up the study of such exotic modes, we recently demonstrated that the DBE and MPC dispersion relations can be replicated using printed microstrip transmission lines and lumped elements [9].

Specifically (see Fig. 1), a pair of transmission lines (consisting of periodic coupled and uncoupled sections) can be used to emulate the MPC and DBE modes. A typical DBE unit cell and its corresponding dispersion are shown in Fig. 2. This simple and easy-to-manufacture model provides means to quickly study, demonstrate, and utilize the

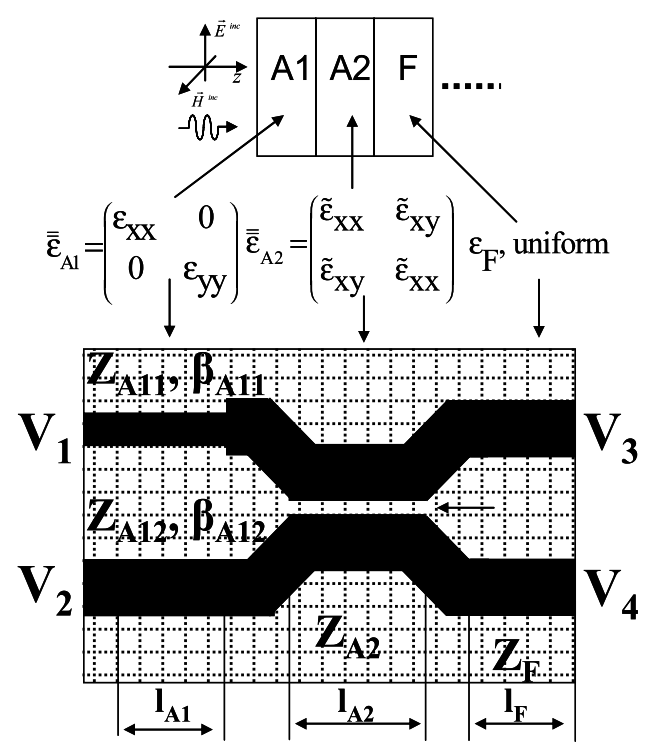

Fig. 1 Coupled lines emulating anisotropy in DBE unit cell structure.

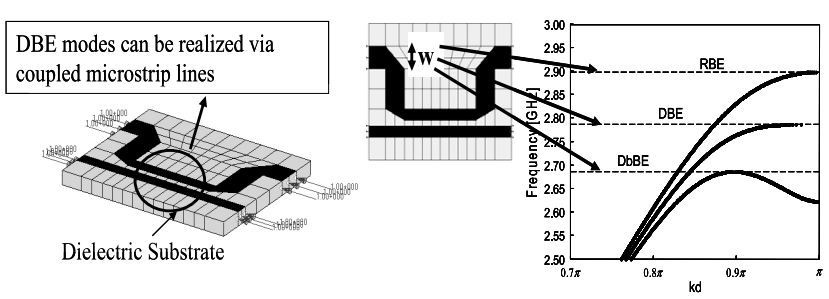

Fig. 2 DBE unit cell structure (left), and corresponding band (k- $\omega)$ diagrams realized by varying the thickness $(w)$ of the indicated microstrip section. 
MPC/DBE modes in engineering applications, such as antennas and microwave components. This paper focuses on one such application, namely a printed antenna structure, utilizing the DBE dispersion.

\section{Exotic Propagation Modes in Degenerate Band Edge and Magnetic Photonic Crystals}

Due to the anisotropic materials forming the MPC/DBE assemblies, the dispersion relation is allowed to have four branches (4 real roots of the characteristic equation) for low frequencies (where the unit cell dimensions are very small as compared to the wavelength). This fourth order system allows for greater design capability when the wavelength is comparable to the unit cell size. Thus, when the operation frequency is close to the band gap, the dispersion relation can be tuned to have a maximally flat edge (corresponding to the DBE diagram having $\omega^{\prime}=0, \omega^{\prime \prime}=0$, and $\omega^{\prime \prime \prime}=0$ ), a regular band edge $\left(\omega^{\prime}=0\right)$, or a double band edge corresponding to a 4th order polynomial behaviour as shown in Fig. 2. Although the crystal does not allow propagation at the band edge frequency, the evanescent waves at the interfaces and the Fabry-Perot resonances of DBE layers allows for the existence of novel modes [7] that can be used to realize novel microwave components and antennas.

\subsection{Frozen Modes in Magnetic Photonic Crystals}

Even more exotic modes can be obtained when a suitable biased ferromagnetic layer is added to the assembly. The presence of the non-reciprocal Faraday rotation layer allows for a more flexible tuning of the 4th order dispersion relation, allowing in turn the occurrence of a stationary inflection point (SIP) with $\omega^{\prime}=0$ and $\omega^{\prime \prime}=0$ as shown in Fig. 3 . Frozen modes supported by such crystals were discussed in [5]. It is important to note that those frozen modes can be excited with little or no reflection since the SIP is achieved within the propagation spectrum, i.e. not in the band gap or at band edges. Utilization of these frozen modes will be the subject of a future paper. Here, we focus on printed antenna concepts using the DBE modes.

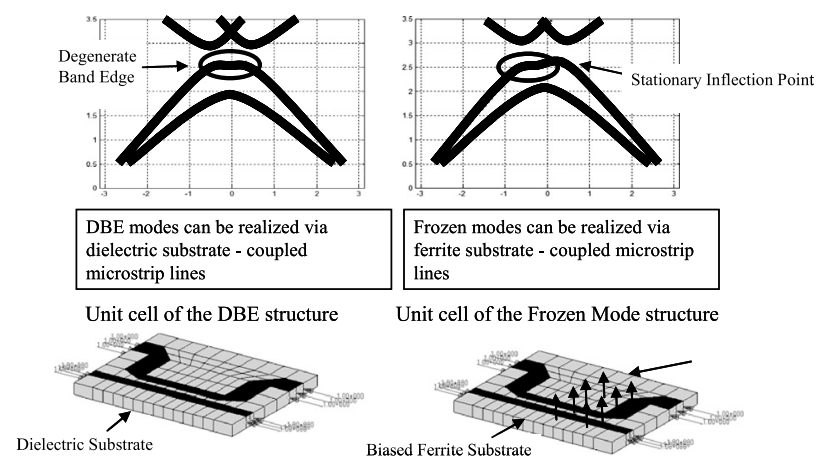

Fig. 3 DBE modes of the coupled microstrip lines (left column) become MPC modes with the proper choice of externally biased ferrite substrate (right column).

\section{Printed Antennas Employing the DBE Dispersion}

The dispersion relation characterizing the periodic material assembly is an abstraction for an infinite medium. When dealing with finite media, resonances due to the material boundaries are inevitable, giving rise to the well known Fabry-Perot resonances and unwanted diffractions from material edges. Also, radiation loss must be taken into account when computing and designing the $\mathrm{k}-\omega$ diagrams of printed microstrip DBE (MS-DBE) structures. The infinite nature of the MS-DBE design must therefore be altered to realize and manufacture a physical antenna. To best utilize the infinite nature of the array (for which a dispersion relation can be formulated), one approach is to cast the unit cells into a circular (cyclic) form such that the fields within the structure see an infinite medium. The number of unit cells must also be kept at a minimum since physically small antennas

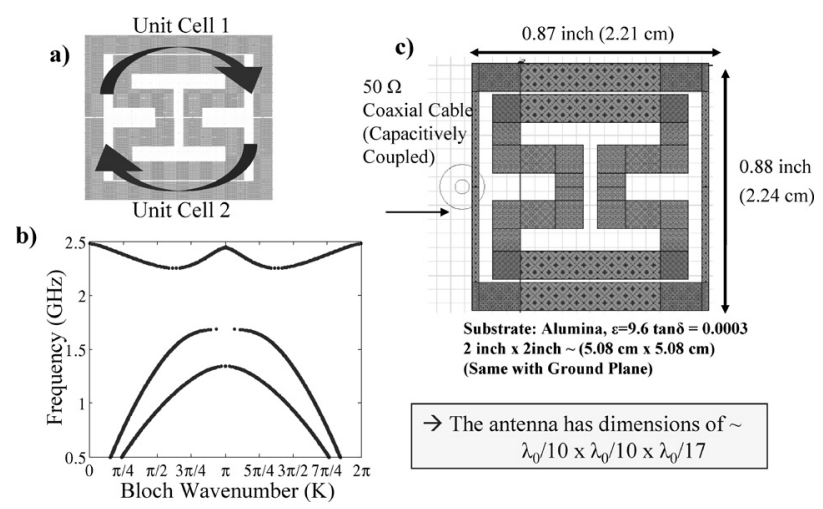

Fig. 4 DBE antenna concept and design example: (a) 2 DBE unit cells wrapped around to form DBE antenna resonator, (b) Dispersion diagram of the DBE unit cell, (c) Dimension of the coaxial fed DBE antenna whose unit cell has the Bloch diagram in (b). The substrate is $\lambda_{o} / 17$ thick and has $\varepsilon_{r}=9.6$ with $\tan \delta \sim 0.0003$.

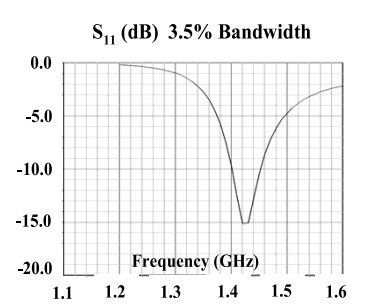

(a)

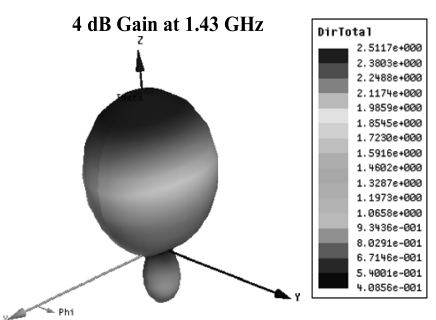

(b)

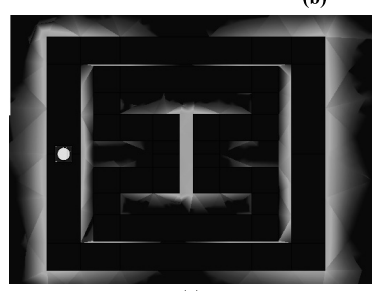

Fig. 5 Performance of the DBE antenna in Fig. 4(c). (a) Return loss matched to a $50 \Omega$ feed at $1.43 \mathrm{GHz}$, (b) Gain pattern at $1.43 \mathrm{GHz}$, (c) Field distribution across the antenna aperture (note that the strong fields are between the center conductors) 
are typically desired. The concept of circular periodicity is displayed in Fig. 4 (upper left) Fig. 4 also gives a prototype design having an alumina $\left(\varepsilon_{r}=9.6, \tan \delta \sim 0.0003\right)$ substrate. As seen, the printed topology consists of two mirror-imaged unit cells that form the circle. This antenna was designed to resonate (i.e. DBE resonance) at $1.43 \mathrm{GHz}$ and is fed by a coaxial line at the uncoupled section. Remarkably, the resulting structure is very small (less that a tenth of the freespace wavelength) in size and has a reasonable bandwidth of $3.5 \%(-10 \mathrm{~dB})$ with a $4 \mathrm{~dB}$ gain (see Fig. 5). To our knowledge, this is among the smallest published antenna elements for the given gain and bandwidth performance.

\section{Phased Array Using DBE Antenna Elements for Beam Steering}

We next proceed to employ the miniature DBE antenna element to construct an array configuration that delivers optimal gain (i.e. near the $\frac{4 \pi A}{\lambda_{o}^{2}}$ where $A$ denotes the array aperture). A key design factor is that the DBE antenna elements exhibit much reduced coupling because the fields are mainly concentrated near its center.

A $4 \times 4$ array configuration is shown in Fig. 6. This array was based on an element design in Fig. 4(c) but was printed on 250 mil. thick Duroid substrate $\left(\varepsilon_{r}=2.2\right.$, $\tan \delta=0.0009$ ), resulting in $3.3 \%$ return bandwidth at $2.525 \mathrm{GHz}$. The same array has a bandwidth of $1.7 \%$ at

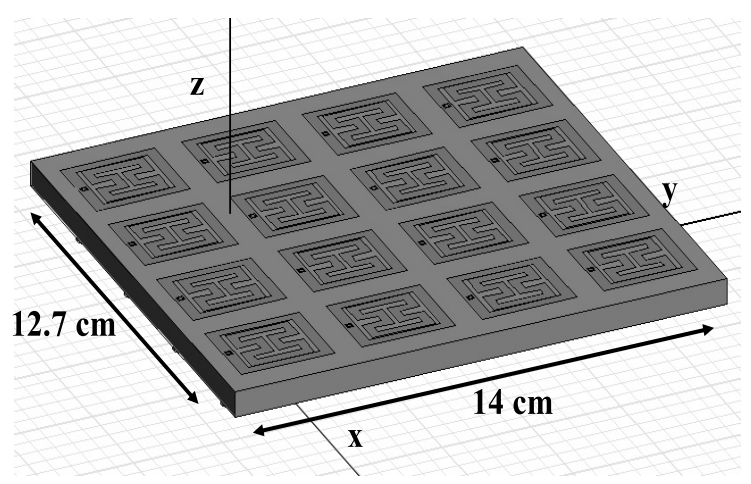

Fig. 616 element DBE antenna array printed on duroid.

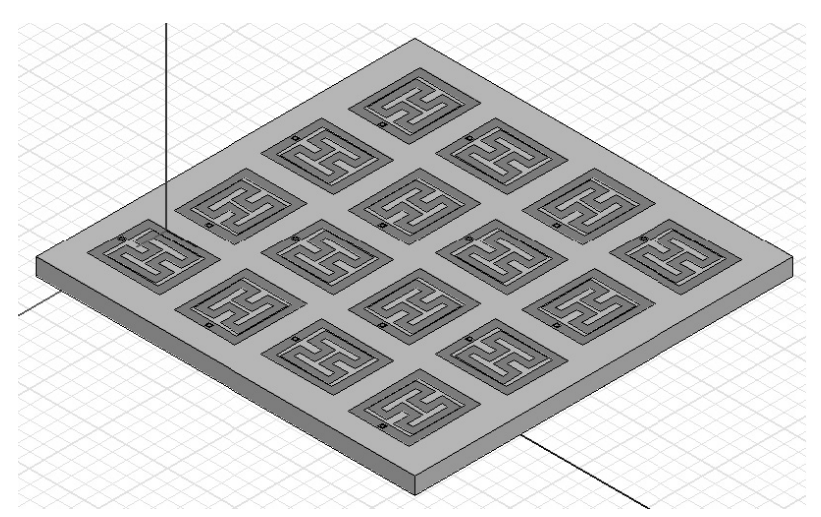

Fig. 716 element dual polarized DBE antenna array.
$2.60 \mathrm{GHz}$ with a broadside directivity of $13 \mathrm{~dB}$ (i.e. near optimum). We remark that the array is slightly larger than a square wavelength $1.1 \lambda_{0} \times 1.2 \lambda_{0}$, and is very thin $\left(\lambda_{0} / 18\right)$. Of importance is that the array beam can be steered down to 80 degrees from broadside with $10 \mathrm{~dB}$ or better gain throughout all scan angles.

As usual, circular and dual polarized arrays can be achieved by a simply rotation every other element in the arrays as shown in Fig. 7. For this configuration, the return loss bandwidth was $2 \%$. But the gain was still maintained at $13 \mathrm{~dB} \mathrm{CP}$ at $2.55 \mathrm{GHz}$.

\section{Remarks and Future Direction}

This paper demonstrated the practical realization of the novel degenerate band-edge modes using a simple printed circuit. The latter was designed to emulate the usual DBE modes by forming a unit cell consisting of a pair of coupled and uncoupled sections of printed microstrip lines. Further, we used circular periodicity to emulate the periodic assembly using a very small region. Specifically, a remarkable small antenna $\lambda_{0} / 10 \times \lambda_{0} / 10 \times \lambda_{0} / 17$ was demonstrated to achieve a gain of $4 \mathrm{~dB}$ with $3.5 \%$ bandwidth. This DBE antenna had the characteristic of concentrating the resonant fields near the center of the antenna elements. Thus, beam steering with better than $10 \mathrm{~dB}$ gain and low sidelobes was achieved down to $80^{\circ}$ from normal.

Perhaps of most importance is that the novel modes (previously demonstrated in actual periodic material assemblies) can now be realized practically overnight using printed circuit $(\mathrm{PCB})$ technologies. Our understanding of the k- $\omega$ diagram (the DNA of the material) is also opening new directions in how to realize new devices, possibly by also introducing lumped elements into the coupled printed circuit unit cell. A conceptual structure supporting DBE/MPC modes and potentially negative refraction is shown in Fig. 8. Today's PCB technology and availability of low loss lumped capacitors and inductors allows for experimentation with structures without a need of costly equipment.
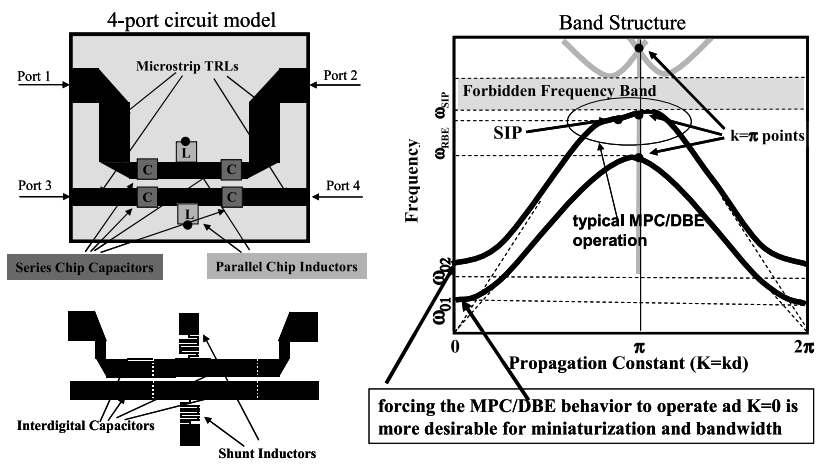

Fig. 8 Concept structure and dispersion characteristics for generating exotic propagation modes in coupled transmission lines loaded with lumped reactive elements. 


\section{Acknowledgements}

This work was supported in part by the Air Force Office of Scientific Research under the MURI grant FA9550-04-10359.

\section{References}

[1] IEEE Trans. Antennas Propag., Special Issue on Metamaterials, vol.51, Oct. 2003.

[2] G.V. Eleftheriades and K.G. Balmain, Negative-Refraction Metamaterials, IEEE Press, John Wiley \& Sons, 2005.

[3] R.F.J. Broas, D.F. Sievenpiper, and E. Yablonovitch, "A high impedance ground plane applied to a cell phone handset geometry," IEEE Trans. Microw. Theory Tech., vol.49, no.7, pp.1262-1265, July 2001.

[4] A. Figotin and I. Vitebskiy, "Nonreciprocal magnetic photonic crystals," Phys. Rev. E, vol.63-066609, pp.1-17, May 2001.

[5] G. Mumcu, K. Sertel, J.L. Volakis, I. Vitebskiy, and A. Figotin, "RF propagation in finite thickness unidirectional magnetic photonic crystals," IEEE Trans. Antennas Propag., vol.53, no.12, pp.4026-4034, Dec. 2005

[6] G. Mumcu, K. Sertel, and J.L. Volakis, "Miniature antennas and arrays embedded within magnetic photonic crystals," IEEE Antennas and Wireless Prop. Lett., vol.5, no.1, pp.168-171, Dec. 2006.

[7] A. Figotin and I. Vitebskiy, "Gigantic transmission band-edge resonance in periodic stacks of anisotropic layers," Phys. Rev. E, vol.72 036619, pp.1-12, Sept. 2005.

[8] S. Yarga, K. Sertel, and J.L. Volakis, "Degenerate band edge crystals and periodic assemblies for high gain antennas," 2006 IEEE APS/URSI/AMEREM Symposium, Albuquerque, NM, July 2006-101.2.

[9] C. Locker, K. Sertel, and J.L. Volakis, "Emulation of propagation in layered anisotropic media with equivalent coupled microstrip lines," IEEE Microwave and Wireless Comp. Lett., vol.16, no.12, pp.642644, Dec. 2006.

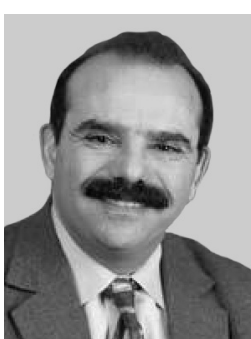

John L. Volakis was born on May 13, 1956 in Chios, Greece and immigrated to the U.S.A. in 1973. He obtained his B.E. Degree, summa cum laude, in 1978 from Youngstown State Univ., Youngstown, Ohio, the M.Sc. in 1979 from the Ohio State Univ., Columbus, Ohio and the Ph.D. degree in 1982, also from the Ohio State Univ. From 1982-1984 he was with Rockwell International, Aircraft Division (now Boeing Phantom Works), Lakewood, CA and during 1978-1982 he was a Graduate Research Associate at the Ohio State University ElectroScience Laboratory. From January 2003 he is the Roy and Lois Chope Chair Professor of Engineering at the Ohio State University, Columbus, Ohio and also serves as the Director of the ElectroScience Laboratory. Prior to moving to the Ohio State Univ., he was a Professor in the Electrical Engineering and Computer Science Dept. at the University of Michigan, Ann Arbor, MI. (1984-2003). He also served as the Director of the Radiation Laboratory from 1998 to 2000. His primary research deals with antennas, computational methods, electromagnetic compatibility and interference, design of new RF materials, multi-physics engineering and bioelectromagnetics. Dr. Volakis published 230 articles in major refereed journal articles ( 9 of these have appeared in reprint volumes), nearly 350 conference papers and 10 book chapters. In addition, he co-authored 3 books: Approximate Boundary Conditions in Electromagnetics (Institution of Electrical Engineers, London, 1995), Finite Element Method for Electromagnetics (IEEE Press, New York, 1998) and Frequency Domain Hybrid Finite Element Methods in Electromagnetics (Morgan \& Claypool). He has also written two well-edited coursepacks on introductory and advanced numerical methods for electromagnetics, and has delivered short courses on numerical methods, antennas and frequency selective surfaces. In 1998 he received the University of Michigan (UM) College of Engineering Research Excellence award and in 2001 he received the UM, Dept. of Electrical Engineering and Computer Science Service Excellence Award. Dr. Volakis is listed by ISI among the top 250 most referenced authors (2004, 2005); He graduated/mentored over $45 \mathrm{Ph} . \mathrm{D}$. students/post-docs, and co-authored with them 5 best paper awards at conferences. Dr. Volakis served as an Associate Editor of the IEEE Transactions on Antennas and Propagation from 1988-1992, and as an Associate Editor of Radio Science from 1994-1997. He chaired the 1993 IEEE Antennas and Propagation Society Symposium and Radio Science Meeting, and co-chaired the same Symposium in 2003. Dr. Volakis was a member of the AdCom for the IEEE Antennas and Propagation Society from 1995 to 1998 and served as the 2004 President of the IEEE Antennas and Propagation Society. He also serves as an associate editor for the J. Electromagnetic Waves and Applications, the IEEE Antennas and Propagation Society Magazine, and the URSI Bulletin. He was elected Fellow of the IEEE in 1996, and is a member of Commissions B and E of URSI.

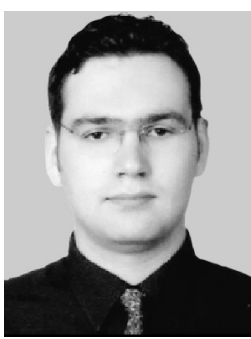

Gokhan Mumcu was born on March 30, 1982 in Bursa, Turkey. He received the B.S degree from Bilkent University, Ankara, Turkey and the M.S. degree from The Ohio State University, Columbus, all in electrical engineering, in 2003 and 2005, respectively. He is currently working toward the Ph.D. degree in the ElectroScience Laboratory, The Ohio State University, as a Graduate Research Associate. His research areas include electromagnetic theory, computational electromagnetics, and metamaterials. 


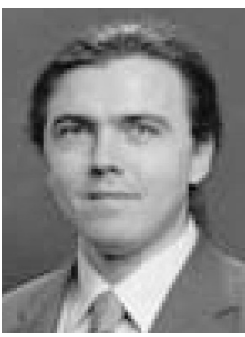

Kubilay Sertel was born on June 27, 1973, in Tekirdag, Turkey. He received the B.S. degree from Middle East Technical University, Ankara, Turkey in 1995, the M.S. degree from Bilkent University, Ankara, Turkey in 1997, and the Ph.D. degree from the Electrical Engineering and Computer Science Department at the University of Michigan, Ann Arbor, MI in 2003, respectively. He is currently a Senior Research Associate at the ElectroScience Laboratory and an Adjunct Assistant Professor at the Electrical and Computer Engineering Department at the Ohio State University. His research areas include electromagnetic theory, computational electromagnetics, volume-surface integral equations and hybrid methods, fast and efficient methods for large-scale electromagnetics problems and parallel implementations of fast algorithms, RF characterization and antenna designs of engineered composite materials. 